\title{
Área y biomasa foliar total de Nothofagus glauca (Phil.) Krasser en zona Andina, Región del Maule, Chile
}

\author{
Total foliar area and biomass of Nothofagus glauca (Phil.) \\ Krasser in Andean area, Maule Region, Chile
}

\section{Óscar Vallejos-Barra ${ }^{\circledR}$, Mauricio Ponce-Donoso ${ }^{2}{ }^{\circledR}$, Pablo Heinrich ${ }^{3}$ y Ursula Doll ${ }^{4}$}

Vallejos-Barra, O., Ponce-Donoso, M., Heinrich, P. y Doll, U. (2019). Área y biomasa foliar total de Nothofagus glauca (Phil.) Krasser en zona Andina, Región del Maule, Chile. Colombia Forestal, 22(2), 5-14.

Recepción: 14 de mayo de 2018

\section{Resumen}

Variables dendrométricas como DAP y altura son usadas para gestionar los bosques, especialmente cuando la especie es endémica y está amenazada. Nothofagus glauca (Hualo) presenta estas características, requiriendo investigación para orientar su manejo sostenible. El objetivo del artículo es ajustar funciones que cuantifiquen el área foliar total (AFT) y biomasa foliar total (BFT) en ejemplares de un bosque relicto ubicado en la Región del Maule (Chile), permitiendo monitorear esta especie. En parcelas de $100 \mathrm{~m}^{2}$ se midió DAP y altura de todos los árboles, apeándose 16 ejemplares, extrayéndoles el total de hojas para determinar su masa fresca. Con muestras de 50 hojas por cada árbol se obtuvo la masa fresca y seca, determinándose el área foliar con LI-COR LI-3100. Usando regresiones se obtuvo significancias estadísticas con área basal, cuyos coeficientes de determinación variaron entre 63.80 y $93.27 \%$, con errores estándar de $8.46 \mathrm{~m}^{2}$ y $0.58 \mathrm{~kg}$ para AFT y BFT, respectivamente. La estimación del índice de área foliar (IAF) bordeó $0.32 \mathrm{~m}^{2} . \mathrm{m}^{-2}$, mientras que para biomasa foliar fue 2.1 ton.ha ${ }^{-1}$.

Palabras clave: área basal, bosque nativo, especie amenazada, funciones dendrométricas, IAF.
Aprobación: 4 de junio de 2019

\begin{abstract}
Dendrometric variables such as DAP and height are used to manage forests, especially when the species is endemic and threatened. Nothofagus glauca (Hualo) presents these characteristics, requiring research to guide its sustainable management. The objective of this article is adjust functions that quantify the total leaf area (TLA) and total leaf biomass (TLB) in specimens of a relict forest located in Maule Region (Chile), allowing to monitor this species. In plots of $100 \mathrm{~m}^{2}, \mathrm{BDH}$ and height of all trees were measured, 16 specimens were removed, extracting the total leaves to determine their fresh mass. With samples of 50 leaves per tree, the fresh and dry mass were obtained, determining the leaf area with LI-COR LI-3100. Using regressions, statistical significance was obtained with a basal area, which its determination coefficients varied between 63.80 and $93.27 \%$, with standard errors of $8.46 \mathrm{~m}^{2}$ and 0.58 $\mathrm{kg}$ for TLA and TLB, respectively. The estimate of the leaf area index (LAI) was $0.32 \mathrm{~m}^{2} . \mathrm{m}^{-2}$, while for leaf biomass it was 2.1 ton.ha ${ }^{-1}$.
\end{abstract}

Key words: basal area, natural forest, threatened species, dendrometric function, LAI.

\footnotetext{
Facultad de Ciencias Forestales, Universidad de Talca. Talca, Chile. ovallejo@utalca.cl. Autor para correspondencia Facultad de Ciencias Forestales, Universidad de Talca. Talca, Chile. mponce@utalca.cl. Forestal Arauco.Valdivia, Chile. pablo.henrich@arauco.cl

Facultad de Ciencias Forestales, Universidad de Talca. Talca, Chile. udoll@utalca.cl
} 


\section{INTRODUCCIÓN}

El ajuste de funciones alométricas en especies forestales, que hagan uso de variables dendrométricas, es ampliamente usado y aceptado para una mejor gestión de los bosques. Entre estas funciones se encuentran las que cuantifican tanto el área y la biomasa foliar, especialmente cuando se trata de especies nativas, puesto que de éstas es posible inferir sobre atributos ecológicos y funcionales de los ecosistemas. En este contexto, Nothofagus glauca (Phil.) Krasser, conocida con los nombres vernáculos de hualo, roble colorado o roble maulino, corresponde a una especie de la familia Nothofagaceae, que se ha adaptado a las condiciones de sequía y altas temperaturas $(\mathrm{Oli}$ vares et al., 2005; Quiroz et al., 2009). En Chile se distribuye en la Cordillera de la Costa entre las provincias de Melipilla y Ñuble, mientras que por la Cordillera de Los Andes, lo hace desde la provincia de Cachapoal hasta el Bío-Bío (Donoso, 1993; Hechenleitner et al., 2005; Muñoz et al., 2018). Como masas boscosas dominadas por N. glauca se ubican entre los ríos Mataquito e Itata por la Cordillera de la Costa y desde el río Maule hasta el Ñuble por la Cordillera de Los Andes (Amigo et al., 2000). Junto a Nothofagus macrocarpa (A. DC.) F.M. Vázquez \& R.A. Rodr. y Nothofagus obliqua (Mirb.) Oerst., constituyen las especies más septentrionales del género en Chile, considerándose en muchas zonas como relicta producto de la última glaciación (Donoso, 1993).

Las poblaciones de $N$. glauca son consideras como vulnerables (IUCN, 2012; Heinrichs et al., 2018) debido a la actual fragmentación producto de sucesivos cambios en el uso del suelo (Le Quesne y Sandoval, 2001), predominando la sustitución vegetal, ya sea por cultivos agrícolas o por plantaciones forestales de especies exóticas de rápido crecimiento (Donoso, 1993; Heinrichs et al., 2016). Por lo anterior, describir estas poblaciones contribuye a su conservación y a una mejor gestión de los bosques.

La disminución de los ecosistemas forestales es de actual interés debido, entre otros aspectos, al papel que tienen en la captura de $\mathrm{CO}_{2}$ (Rojo et al., 2003), considerando que las emisiones de este gas aumentaron en cerca de un $80 \%$ entre 1970 y 2004 (IPCC, 2007). Las plantas utilizan $\mathrm{CO}_{2}$ y liberan $\mathrm{O}_{2}$ a la atmósfera mediante la fotosíntesis, y a diferencia de las especies anuales, los árboles crecen almacenando los fotoasimilados durante periodos prolongados de tiempo en sus estructuras leñosas (Acosta et al., 2002). Entre otras condiciones, el crecimiento de los árboles y plantas dependen de la cantidad de luz interceptada por su follaje (Beadle, Honeysettt, Turnbull y White, 1995), razón por la cual el área o biomasa foliar en plantaciones agrícolas y forestales es fundamental para evaluar su estado de nutrición (Ruiz et al., 2007), predecir el crecimiento (Wanga et al., 2019), rendimiento frutal (Lepe, Yuri y Moggia, 2005; Acosta et al., 2008), rendimiento maderable, absorción de carbono (Rodríguez et al., 2011), tasa de transpiración (Simonin et al., 2006), aporte de hojarasca al suelo (Aceñolaza et al., 2009), eficiencia en el uso de agua y conversión de fotoasimilados (Cabezas et al., 2008), entre otras.

A partir del área foliar se obtiene el índice de área foliar (IAF), variable ampliamente utilizada por su asociación con los distintos factores de producción vegetal (De la Casa et al., 2007), usado además como indicador de procesos fisiológicos, como fotosíntesis, transpiración y evaporación (Moser et al., 2007; Olivas et al., 2013) que para Cabezas et al. (2008), constituye uno de los elementos más importantes para el estudio de bosques y plantaciones forestales (Moser et al., 2007; Olivas et al., 2013), pudiendo determinar incluso la cantidad de erosión del suelo (Lin et al., 2019). Sin embargo, son aún escasos los modelos para estimar área foliar y biomasa foliar en especies leñosas nativas de Chile, encontrándose los modelos de 
BFT de Aguirre y Infante (1988) para Peumus boldus Mol. y Acacia caven (Mol.) Mol., Gyenge et al., (2009) para Nothofagus antarctica (Forst.) Oerst., Schinus patagonicus (Phil.) I.M. Johnst. ex Cabr. y para Lomatia hirsuta (Lam.) Diels ex J.F. Macbr. y Dobbs, Hernández y Escobedo (2011) para Schinus molle Raddi. y Quillaja saponaria Mol. También Dobbs et al. (2011) presentan un modelo para el AFT de Maytenus boaria Mol, no habiendo estudios específicos para Nothofagus glauca.

Distintos estudios han demostrado la relación existente entre el área foliar y biomasa foliar con el diámetro a la altura del pecho, diámetro a la altura del tocón, área basal o área de albura (Ganskopp y Miller, 1986; Barrantes y Garcia, 1989; Nowak, 1996; Simonin et al, 2006; Calvo, McDowell y Waring, 2008; Fonseca, Alice y Rey, 2009; Gyenge et al., 2009; Ladesma, Carranza y Balzarini, 2010; Dobbs et al., 2011; Rodríguez et al., 2011; Rubilar et al., 2012) entre todos ellos, la variable que mejor explica esta relación es el área de albura, que corresponde a la parte activa del xilema que transporta agua y nutrientes (Rodríguez et al., 2011). Sin embargo, determinar el área de albura resulta complejo dado que es necesario extraer rodelas $u$ obtener muestras usando un taladro de incremento. Al respecto, Cano (1993) afirma que en árboles jóvenes es posible relacionar el área foliar con el diámetro a la altura del pecho, puesto que la porción del duramen es mínima.

En el contexto descrito, el objetivo de este trabajo fue ajustar funciones que cuantifiquen área y biomasa foliar de la especie Nothofagus glauca, utilizando como variables explicativas el diámetro a la altura del pecho, la altura total del árbol y combinaciones de ellas.

\section{MATERIALES Y MÉTODOS}

Los árboles que se utilizaron se ubicaban en el Centro Experimental "El Picazo" de la Universi-

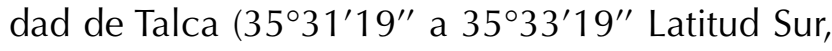

$71^{\circ} 08^{\prime} 45^{\prime \prime}$ a $71^{\circ} 12^{\prime} 49^{\prime \prime}$ Longitud Oeste), localizado en la comuna de San Clemente, provincia de Talca, zona precordillera andina de la región del Maule, Chile. El Centro Experimental tiene una superficie de 1,422 ha, con 574 ha de renoval de bosque nativo del tipo forestal roble-hualo (Arriagada, 2004). Las características edafoclimáticas señalan que el suelo está constituido por derivados de material volcánico (Andisol), de pedregosidad moderada, textura franca a franco-arcillosa, delgado y con presencia de erosión moderada producto del viento y el agua. El clima es templado-cálido con estación seca prolongada, Csb en la clasificación Köppen-Geiger. La temperatura media mínima invernal bordea los $2^{\circ} \mathrm{C}$ y las lluvias pueden alcanzar los 1,200 $\mathrm{mm}$ anuales, mientras que en periodo estival la temperatura media máxima alcanza los $27^{\circ} \mathrm{C}$ (Arriagada, 2004).

Selección de árboles y registro de variables. En un único rodal de 574 ha, multietáneo, se establecieron tres parcelas de $100 \mathrm{~m}^{2}$, encontrándose en todas ellas un total de 50 especímenes de N. glauca, definiéndose seis clases diamétricas entre 5 y 30 cm, cuya equivalencia alcanza a los 1,666 árboles. ha-1 y un área basal de $38.07 \mathrm{~m}^{2}$.ha-1 (Tabla 1). Se seleccionaron 16 individuos al azar para realizar el estudio, midiéndoles las variables dendrométricas de diámetro a la altura del pecho (DAP, en $\mathrm{cm}$ ) utilizando una forcípula Haglof con precisión al mm y altura total (h, en m) con un clinómetro Suunto PM-5 con precisión de $50 \mathrm{~cm}$. Una vez apeados los árboles se recolectó la totalidad de sus hojas y se registró su masa fresca usando una balanza analítica Excell modelo Unicell con precisión de $1 \mathrm{~g}$. Pese al número limitado de árboles muestreados, la existencia de individuos en las diferentes clases diamétricas estudiadas, permitió asegurar la representatividad de los datos, un aspecto similar se observó en otros estudios (Calvo et al., 2008; Gyenge et al., 2009; Dobbs et al., 2011; Díaz et al., 2011; Timilsina et al., 2017; Sillett et al., 2019). Lo anterior tiene relevancia dado que la utilización de métodos destructivos para la obtención de datos, 
como es este caso, debe procurar la conservación de las especies, especialmente como la estudiada, para lo cual se presentó un plan de corta al servicio forestal, denominado Corporación Nacional Forestal, el que fue aprobado

Por restricción de la estufa secadora, se extrajo al azar una muestra de 50 hojas por árbol estudiado, registrándose su masa fresca y luego seca. Se midió el área foliar de las muestras mediante el LI-COR LI3100. El área foliar total por árbol (AFT) se expresó en $\mathrm{m}^{2}$ y se determinó mediante la ecuación 1.

$$
A F T_{i}=\frac{A F M_{i}}{M F M_{i}} * M F T_{i}
$$

Donde:

$A F T_{\mathrm{i}}$ = área foliar total del árbol i, en $\mathrm{m}^{2}$

$A F M_{\mathrm{i}}=$ área foliar de la muestra de hojas del árbol i, en $\mathrm{m}^{2}$

$M F M_{\mathrm{i}}=$ masa fresca de la muestra de hojas del árbol i, en kg

$M F T_{\mathrm{i}}=$ masa fresca del total de hojas del árbol i, en $\mathrm{kg}$

Para determinar la biomasa foliar total por árbol (BFT) expresado en toneladas, se utilizaron las hojas muestreadas, calculando la masa seca, de acuerdo a la ecuación 2.

$$
B F T_{i}=\frac{M S M_{i}}{M F M_{i}} * M F T_{i}
$$

Donde:

$B F T_{\mathrm{i}}=$ biomasa foliar total del árbol i, en kg

$M S M_{\mathrm{i}}=$ masa seca de la muestra de hojas del árbol i, en kg

$M F M_{\mathrm{i}}=$ masa fresca de la muestra de hojas i, en $\mathrm{kg}$

$M F T_{\mathrm{i}}=$ masa fresca del total de hojas del árbol i, en $\mathrm{kg}$

Ajuste, selección de modelos y estimaciones. Se observaron las relaciones de las variables AFT y BFT con las variables dendrométricas medidas, esto es DAP y $h$, además de sus transformaciones. Se ajustaron modelos de regresión del tipo lineal simple (método de los mínimos cuadrados) y no lineal (método de estimación de Marquardt), representados por las ecuaciones 3 y 4 , respectivamente.

$$
\begin{gathered}
Y=\beta_{0}+\beta_{1} * X+\varepsilon \\
Y=\beta_{0} * X^{\beta_{1}}+\varepsilon
\end{gathered}
$$

Donde:

$Y=$ variable dependiente del modelo (en este caso AFT o BFT)

$\beta_{0}, \beta_{1}=$ parámetros de los modelos

$X=$ variable independiente del modelo

$\mathrm{e}=$ error del modelo (media 0; varianza $\sigma^{2}$ )

Para seleccionar el modelo propuesto, se determinó la significancia de los coeficientes, el coeficiente de determinación $\left(R^{2}\right)$, el error cuadrático medio (ECM) y el criterio de información de Akaike (AIC).

El valor del AIC es una medida que valora la pérdida de información que conlleva la utilización de una función (Mazerolle, 2006). Aunque por sí mismo su valor no es relevante, dado que lo importante es obtener la diferencia del AIC entre cada función ajustada y la función con el menor valor (Burnham et al., 2011), así la mejor función tendrá una diferencia igual a cero; si la diferencia es hasta 2, la función podría ser igualmente satisfactoria; pero una diferencia entre 4 y 7 reduce la significancia de la función, mientras que una diferencia mayor a 10 descarta la utilización de la función.

Con los modelos seleccionados y validados se realizó una estimación por hectárea de los datos recolectados, cuantificando las variables por unidad de superficie.

Los datos fueron procesados en el programa Microsoft Excel ${ }^{\circledR} 2016$ y Statgraphics Centurion $®$, versión XVI.

\section{RESULTADOS}

La tabla de rodal que caracteriza el rodal muestreado y las estimaciones de IAF y BFT corresponde al promedio de los modelos testeados (tabla 1). 
Para las clases diamétricas de 10 a $30 \mathrm{~cm}$ se usaron 3 árboles, mientras que para la de $5 \mathrm{~cm}$ se utilizó un único árbol.

Relación entre las variables. Para los 16 individuos seleccionados el AFT varió entre 9.73 y 56.1 $\mathrm{m}^{2}$, en tanto el BFT entre 0.52 y $4.11 \mathrm{~kg}$. La matriz de correlación entre las variables (Figura 1), muestra que el área basal (g) presentó la mayor correlación tanto con la variable AFT $(r=0.8229)$ como BFT $(r=0.8551)$, superando al DAP y sus transformaciones. La altura ( $h$ ) no tuvo relación con AFT y BFT con $r=0.0472$ y $r=0.0128$, respectivamente; además, al combinarse con el DAP la relación decreció en ambas variables dependientes. En consideración a lo anterior, el ajuste se realizó haciendo uso del área basal.
Modelos de área foliar y biomasa foliar ajustados. En los modelos ajustados (tabla 2), AFT 1 y $\mathrm{BFT}_{1}$ el estadígrafo del parámetro $\beta_{0}$ no fue significativo $(p>0.05)$ y al eliminarse este parámetro se obtuvo modelos con menor $\mathrm{AIC}\left(\mathrm{AFT}_{2}\right.$ y $\left.\mathrm{BFT}_{2}\right)$, aunque presentó un mayor EEE. Los otros modelos $\left(\mathrm{AFT}_{3}\right.$ y $\mathrm{BFT}_{3}$ ) obtuvieron una diferencia de AIC cercano al valor 2, de modo que también podrían ser utilizados para la estimación del AFT o el BFT.

A partir de los resultados obtenidos, en cada una de las tres parcelas se realizó una estimación por hectárea, amplificando el valor encontrado en cada una, tanto para IAF como BFT (tabla 3). Las estimaciones fueron muy similares, aunque la estimación con el modelo lineal sin el parámetro $\beta_{0}$ es la más conservadora; no obstante, tiene el mayor error muestral porcentual (28.2\%).

Tabla 1. Tabla de rodal y estimación promedio del IAF y BFT

\begin{tabular}{|c|c|c|c|c|c|c|c|c|}
\hline \multirow{2}{*}{$\begin{array}{c}\begin{array}{c}\text { DAP } \\
(\mathbf{c m})\end{array} \\
5\end{array}$} & \multirow{2}{*}{$\begin{array}{c}\begin{array}{c}\text { Número de árboles } \\
\left(\mathbf{N}^{\circ} . \mathbf{h a}^{-1}\right)\end{array} \\
33.3\end{array}$} & \multirow{2}{*}{$\begin{array}{c}\begin{array}{c}\text { Área basal } \\
\left(\mathbf{m}^{2} . \mathbf{h a}^{-1}\right)\end{array} \\
0.1072\end{array}$} & \multicolumn{3}{|c|}{$\begin{array}{c}\text { IAF } \\
\left(\mathbf{m}^{2} \cdot \mathbf{m}^{-2}\right)\end{array}$} & \multicolumn{3}{|c|}{$\begin{array}{c}\text { BFT } \\
\left(\text { Ton.ha' }^{-1}\right)\end{array}$} \\
\hline & & & 0.02 & \pm & 0.02 & 0.01 & \pm & 0.00 \\
\hline 10 & 400.0 & 3.4583 & 0.36 & \pm & 0.17 & 0.21 & \pm & 0.05 \\
\hline 15 & 666.7 & 11.4797 & 1.02 & \pm & 0.21 & 0.66 & \pm & 0.06 \\
\hline 20 & 400.0 & 12.3315 & 0.99 & \pm & 0.07 & 0.68 & \pm & 0.02 \\
\hline 25 & 66.7 & 3.2527 & 0.25 & \pm & 0.01 & 0.18 & \pm & 0.00 \\
\hline 30 & 100.0 & 7.4354 & 0.54 & \pm & 0.04 & 0.40 & \pm & 0.01 \\
\hline Total & $1,666.7$ & 38.0648 & 3.18 & \pm & 0.52 & 2.13 & \pm & 0.11 \\
\hline
\end{tabular}

\begin{tabular}{|c|c|c|c|c|c|c|c|}
\hline $\begin{array}{l}\text { AFT } \\
-\square+\end{array}$ & ${ }_{0} \operatorname{sog}^{8^{2}}$ & 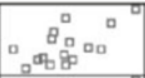 & 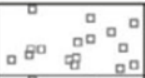 & 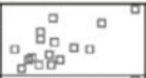 & 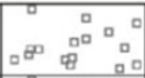 & 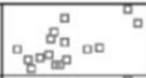 & $a^{4 a^{4}}$ \\
\hline $\begin{array}{l}0.9599 \\
\mathbf{0 . 0 0 0 0}\end{array}$ & BFT & 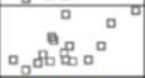 & 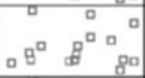 & a & 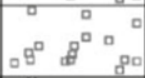 & 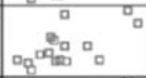 & वृ \\
\hline $\begin{array}{l}0.6073 \\
0.0126\end{array}$ & $\begin{array}{l}0.6478 \\
\mathbf{0 . 0 0 6 7}\end{array}$ & $\begin{array}{l}\text { DAP } \\
\square=1\end{array}$ & $\begin{array}{ll}0 \\
0\end{array}$ & & 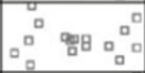 & 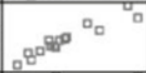 & $\overline{0}$ \\
\hline $\begin{array}{l}0.0472 \\
\mathbf{0 . 8 6 2 3}\end{array}$ & $\begin{array}{l}0.0128 \\
0.9626\end{array}$ & $\begin{array}{l}0.0240 \\
\mathbf{0 . 9 2 9 8}\end{array}$ & $\frac{\mathbf{h}}{-t}$ & 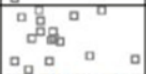 & $a^{a^{4}}$ & $\begin{array}{cccc}0 & 0 & 0 \\
0 & 0 \\
0 & 0 & 0 \\
0 & 0 & 0\end{array}$ & 8 \\
\hline $\begin{array}{l}0.6356 \\
\mathbf{0 . 0 0 8 1}\end{array}$ & $\begin{array}{l}0.6647 \\
\mathbf{0 . 0 0 5 0}\end{array}$ & $\begin{array}{l}0.9876 \\
\mathbf{0 . 0 0 0 0}\end{array}$ & $\begin{array}{c}-0.0250 \\
\mathbf{0 . 9 2 6 9}\end{array}$ & $\begin{array}{l}\text { DAP^2 }^{\prime} \\
-U\end{array}$ & 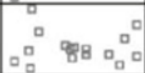 & 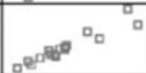 & 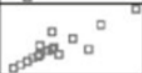 \\
\hline $\begin{array}{l}0.0428 \\
\mathbf{0 . 8 7 4 8}\end{array}$ & $\begin{array}{l}0.0053 \\
\mathbf{0 . 9 8 4 5}\end{array}$ & $\begin{array}{l}0.0247 \\
\mathbf{0 . 9 2 7 7}\end{array}$ & $\begin{array}{l}0.9981 \\
\mathbf{0 . 0 0 0 0}\end{array}$ & $\begin{array}{c}-0.0207 \\
\mathbf{0 . 9 3 9 3}\end{array}$ & $\mathrm{h}^{\wedge} \mathbf{2}$ & \begin{tabular}{|ccc}
0 & 0 & \\
0 \\
0 \\
0 \\
0
\end{tabular} & $p^{\circ}$ \\
\hline $\begin{array}{l}0.6308 \\
\mathbf{0 . 0 0 8 8}\end{array}$ & $\begin{array}{l}0.6373 \\
\mathbf{0 . 0 0 7 9}\end{array}$ & $\begin{array}{l}0.9535 \\
\mathbf{0 . 0 0 0 0}\end{array}$ & $\begin{array}{l}0.2491 \\
\mathbf{0 . 3 5 2 2}\end{array}$ & $\begin{array}{l}0.9488 \\
\mathbf{0 . 0 0 0 0}\end{array}$ & $\begin{array}{l}0.2559 \\
\mathbf{0 . 3 3 8 8}\end{array}$ & $\begin{array}{c}\mathbf{D A P}^{\wedge} \mathbf{2}^{*} \mathbf{h} \\
-\square+\end{array}$ & \\
\hline $\begin{array}{l}0.8229 \\
\mathbf{0 . 0 0 0 1}\end{array}$ & $\begin{array}{l}0.8551 \\
\mathbf{0 . 0 0 0 0}\end{array}$ & $\begin{array}{l}0.8780 \\
\mathbf{0 . 0 0 0 0}\end{array}$ & $\begin{array}{l}0.0909 \\
\mathbf{0 . 7 3 7 7}\end{array}$ & $\begin{array}{l}0.8874 \\
\mathbf{0 . 0 0 0 0}\end{array}$ & $\begin{array}{l}0.0917 \\
\mathbf{0 . 7 3 5 4}\end{array}$ & $\begin{array}{l}0.8752 \\
\mathbf{0 . 0 0 0 0}\end{array}$ & -4 \\
\hline
\end{tabular}

Figura 1. Matriz de correlación entre variables. La correlación y la probabilidad se presentan de manera numérica; la correlación se ubica en la parte superior de la cuadrícula y la probabilidad, en la parte inferior, destacada en negrita 
Tabla 2. Modelos ajustados para la estimación de área foliar (AFT, en $\mathrm{m}^{2}$ ) y biomasa foliar total (BFT, en $\mathrm{kg}$ ) usando el área basal (g) e indicadores de calidad de ajuste

\begin{tabular}{lllllll}
\hline \multicolumn{1}{c}{ Modelo ajustado } & $\begin{array}{c}\text { Prob } \\
\boldsymbol{\beta}_{\mathbf{0}}\end{array}$ & Prob $\boldsymbol{\beta}_{\mathbf{1}}$ & $\mathbf{R}^{\mathbf{2}} \mathbf{( \% )}$ & EEE & AIC & D AIC \\
\hline $\mathrm{AFT}_{1}=5.9599382+629.5741791 \mathrm{~g}$ & 0.198 & 0.001 & 65.40 & 8.325 & 33.45 & 1.62 \\
$\mathrm{AFT}_{2}=768.1384739 \mathrm{~g}$ & - & 0.001 & 92.52 & 8.552 & 31.83 & 0.00 \\
$\mathrm{AFT}_{3}=452.13595 \mathrm{~g} 0.8264509751$ & 0.006 & 0.001 & 63.80 & 8.515 & 33.77 & 1.94 \\
$\mathrm{BFT}_{1}=0.16735669+50.39425473 \mathrm{~g}$ & 0.597 & 0.001 & 71.20 & 0.585 & -3.45 & 2.33 \\
$\mathrm{BFT}_{2}=54.28517776 \mathrm{~g}$ & - & 0.001 & 93.27 & 0.571 & -5.79 & 0.00 \\
$\mathrm{BFT}_{3}=44.580438 \mathrm{~g} 0.9352416902$ & 0.005 & 0.001 & 70.95 & 0.588 & -3.38 & 2.40 \\
\hline
\end{tabular}

Tabla 3. Estimación del índice de área foliar (IAF) y biomasa foliar total (BFT) según modelo

\begin{tabular}{|c|c|c|c|c|c|c|}
\hline \multirow{2}{*}{ Parcelas } & $\mathrm{IAF}_{1}$ & $\mathrm{IAF}_{2}$ & $\mathrm{IAF}_{3}$ & $\mathrm{BFT}_{1}$ & $\mathrm{BFT}_{2}$ & $\mathrm{BFT}_{3}$ \\
\hline & \multicolumn{3}{|c|}{$\left(m^{2} \cdot m^{-2}\right)$} & \multicolumn{3}{|c|}{ (Ton.ha $\left.{ }^{-1}\right)$} \\
\hline 1 & 0.40 & 0.31 & 0.37 & 2.45 & 2.21 & 2.35 \\
\hline 2 & 0.22 & 0.18 & 0.21 & 1.39 & 1.30 & 1.36 \\
\hline 3 & 0.40 & 0.38 & 0.39 & 2.74 & 2.69 & 2.71 \\
\hline Promedio & 0.34 & 0.29 & 0.32 & 2.20 & 2.07 & 2.14 \\
\hline Error muestral & 0.087 & 0.082 & 0.082 & 0.58 & 0.58 & 0.58 \\
\hline Error muestral \% & 25.90 & 28.20 & 25.60 & 26.80 & 28.20 & 27.00 \\
\hline
\end{tabular}

\section{DISCUSIÓN}

Los indicadores de ajuste de los modelos estimados de AFT y BFT, resultaron similares a los reportados por Nowak (1996), Calvo et al. (2008), Fonseca et al. (2009), Gyenge et al. (2009), Dobbs et al. (2011) y Timilsina et al. (2017), aunque inferiores a los obtenidos por Sillett et al. (2019). Para este estudio la variable independiente que mejor modela es el área basal (figura 1), a diferencia de estudios citados precedentemente, cuya variable independiente fue el DAP. Otros estudios señalan obtener ajustes adecuados a través del uso de variables independientes como el diámetro del fuste a los $15 \mathrm{~cm}$ del suelo, diámetro de ramas gruesas (mayores a $5 \mathrm{~cm}$ ), diámetros de copa, factores de sombreado, altura de inicio de copa, altura total y área de albura, entre otras, para estimar el AFT y BFT (Ganskopp y Miller, 1986; Barrantes y García, 1989; Simonin et al., 2006; Ladesma et al., 2010; Rodríguez et al., 2011).

Considerando que la altura (h) no se relacionó significativamente con los componentes foliares estudiados, es posible señalar que en la modelación del AFT y BFT se requiere establecer la o las variables que mejor las describen, por cuanto no es posible generalizar respecto de una variable dendrométrica (Ganskopp y Miller, 1986; Barrantes y García, 1989; Simonin et al., 2006; Ladesma et al., 2010; Rodríguez et al., 2011; Timilsina et al., 2017).

Al relacionar la información contenida en la tabla 3, fue posible determinar la cantidad de área foliar requerida para obtener un kilo de biomasa foliar. Para este caso, en términos promedios, se requieren $0.149 \mathrm{~m}^{2}$ de área foliar por gramo de biomasa foliar. Este valor difiere a lo reportado por Sillett et al. (2019) para Sequoia sempervirens, con un valor promedio de $0.056 \mathrm{~m}^{2} \cdot \mathrm{gr}^{-1}$, lo cual indica con claridad que la morfología foliar es particular de cada especie o género en estudio.

Por su parte, en estudios realizados en la Patagonia argentina mediante teledetección, se determinaron valores de IAF entre 0.2 a $2.6 \mathrm{~m}^{2} . \mathrm{m}^{-2}$ para bosques primario y de regeneración de $\mathrm{No}^{-}$ thofagus pumilio (Martínez et al., 2008) similar valor inferior al hallado en este estudio y muy por 
debajo del reporte de $1.57 \pm 0.52 \mathrm{~m}^{2} . \mathrm{m}^{-2}$ (Díaz et al., 2011). Los valores obtenidos del IAF en este estudio, que bordean los $0.32 \mathrm{~m}^{2} . \mathrm{m}^{-2}$, dan cuenta que se trabajó con renovales de bosques secundarios, ya que, si se comparan con bosques de conífera, cuyo valor alcanza rangos de 1.0 a $6.0 \mathrm{~m}^{2} . \mathrm{m}^{-2}$ (Oztürk, Bolat y Ergun, 2015).

\section{CONCLUSIÓN}

El ajuste de funciones que consideran el área basal del árbol como variable explicativa describió con mayor significancia el área foliar total (AFT) y biomasa foliar total (BFT) para la especie Nothofagus glauca ubicado en la precordillera andina de la Región del Maule, Chile.

Los modelos lineales sin intercepto $\left(\mathrm{AFT}_{2}\right.$ y $\left.\mathrm{BFT}_{2}\right)$ presentaron los menores AIC por lo que son los modelos recomendados para estimar estas variables.

\section{CONFLICTO DE INTERESES}

Los autores declaran no tener conflicto de intereses.

\section{CONTRIBUCIÓN POR AUTOR}

O. V-B, U.D. idearon la investigación; O. V-B y P.H. Ilevaron a cabo la investigación en terreno; O. V-B. y M. P-D, analizaron los datos y redactaron el manuscrito. Todos los autores contribuyeron a la discusión y comentaron los borradores.

\section{AGRADECIMIENTOS}

Los autores agradecen al Fondo de Protección Ambiental del Ministerio del Medio Ambiente de Chile por financiar el proyecto 07-003-2009: "Análisis temporal de la biomasa acumulada y carbono absorbido aéreo y radicular, en un renoval de Nothofagus glauca".

\section{REFERENCIAS}

Aceñolaza P., Zamboni, L. y Gallardo, J. (2009). Aporte de hojarasca en bosques del predelta del río Paraná (Argentina). Bosque, 30(3), 135-145.

http://doi.org/10.4067/S0717-92002009000300003

Acosta, E., Acosta, J., Amador, M. y Padilla, J. (2008). Relación entre índice de área foliar y rendimiento en frijol bajo condiciones de secano. Agricultura Técnica en México, 34(1), 13-20. Recuperado de

http://www.scielo.org.mx/scielo.php?script=sci_arttext\&pid=S0568-25172008000100002

Acosta M., Vargas, J., Velázquez, A. y Etchevers, J. (2002). Estimación de la biomasa aérea mediante el uso de relaciones alométricas en seis especies arbóreas en Oaxaca, México. Agrociencia, 36(6), 725-736. Recuperado de

https://www.colpos.mx/agrocien/Bimestral/2002/novdic/art-10.pdf

Aguirre, S. y Infante, P. (1988). Funciones de biomasa para boldo (Peumus boldus) y espino (Acacia caven) de la zona central de Chile. Ciencia e Investigación Forestal, 2(1), 45-50. Recuperado de

http://bibliotecadigital.ciren.cl/bitstream/handle/123456789/10683/ \%28353 \%29-Revista \%20CIFOR \%20volumen \%2015 \%20n \%C2 \%B03 \%2C \%202009 \%20parte \%202. pdf? sequence $=1$ \&isAllowed $=y$

Amigo, J., San Martín, J. y García, L. (2000). Estudio fitosociológico de los bosques de Nothofagus glauca (Phil.) Krasser del Centro-Sur de Chile. Phytocoenología, 32(2), 193-221.

https://doi.org/10.1127/phyto/30/2000/193

Arriagada, P. (2004). Evaluación de impacto ambiental del plan de actividades de la Estación Experimental "El Picazo". Memoria Ingeniería Forestal. Talca, Chile. Universidad de Talca. Recuperado de http://dspace.utalca.cl/handle/1950/1334

Barrantes, O. y García, C. (1989). Estimación de área foliar a partir de la superficie de albura en Pinus sylvestris. Options Méditerranéennes, Serie Séminaires, 3, 53-56. Recuperado de

http://om.ciheam.org/article.php?IDPDF=Cl000505 
Beadle C.L., Honeysett, J.L., Turnbull, C. y White, D. (1995). Site limits to achieving genetic potential. En: B. Potts, N. Borralho, J. Reid, R. Cromer, W. Tibbits y C. Raymond (eds.), Eucalypt Plantations: Improving Fibre Yield and Quality. Proceedings CRC Temperate Hardwood Forestry-IUFRO Conference. Hobart, Australia.

Burnham, K.P., Anderson, D.R. y Huyvaert, K.P. (2011). AIC model selection and multimodel inference in behavioral ecology: some background, observations, and comparisons Behavioral Ecological Sociobiology, 65, 23-35.

https://doi.org/10.1007/s00265-010-1029-6

Cabezas, M., Peña, F., Díaz, C. y Moreno, A. (2008). Dosel de tres especies forestales y su relación con la adaptación a suelos degradados por la erosión. Revista U.D.C.A Actualidad \& Divulgación Científica, 11(2), 175-185. Recuperado de

https://revistas.udca.edu.co/index.php/ruadc/article / view/634

Calvo, J., McDowell, N. y Waring, R. (2008). Allometric relationships predicting foliar biomass and leaf area: sapwood area ratio from tree height in five Costa Rican rain forest species. Tree Physiology, 28, 1601-1608.

https://doi.org/10.1093/treephys/28.11.1601

Cano, E. (1993). Relación alométrica entre el área foliar y el área de la albura en Pinus patula Schl. et Cham., en Zacatlán, Puebla (tesis Maestría en Ciencias). Montecillo, México. Colegio de Posgraduados.

De la Casa, A., Ovando, G., Bressanini, L., Rodríguez, A. y Martínez, J. (2007). Uso del índice de área foliar y del porcentaje de cobertura del suelo para estimar la radiación interceptada en papa. Agricultura Técnica, 67(1), 78-85.

https://doi.org/10.4067/S0365-28072007000100010

Díaz, G.M., Mohr-Bell, D., Lencias, J.D., Menger, M. y Del Valle, H. F. (2011). Teledección satelital cuantitativa para estimar el área basal del bosque de Nothofagis pumilio (Nothogagaceae): el rol del índice de área foliar como información auxiliar. Revista Chilena de Historia Natural, 84(4), 509-521. Recuperado de https://scielo.conicyt.cl/pdf/rchnat/v84n4/art04.pdf
Dobbs C., Hernández, J. y Escobedo, F.J. (2011). Above ground biomass and leaf area models based on a non destructive method for urban trees of two communes in Central Chile. Bosque, 32(3), 287-296.

https://doi.org/10.4067/S0717-92002011000300010

Donoso, C. (1993). Bosques templados de Chile y Argentina. Variación, estructura y dinámica. Santiago, Chile: Editorial Universitaria.

Fonseca, W., Alice, F. y Rey, J. (2009). Modelos para estimar la biomasa de especies nativas en plantaciones y bosques secundarios en la zona Caribe de Costa Rica. Bosque, 30(1), 36-47.

https://doi.org/10.4067/S0717-92002009000100006

Ganskopp, D. y Miller, R. (1986). Estimating leaf area of big sagebrush from measurement of sapwood. Journal of Range Management, 30(4), 338-340.

https://doi.org/10.2307/3899775

Gyenge J., Fernández, M., Sarasola, M., de Urquiza, M. y Schlichter, T. (2009). Ecuaciones para la estimación de biomasa aérea y volumen de fuste de algunas especies leñosas nativas en el valle del río Foyel, NO de la Patagonia argentina. Bosque, 30(2), 95-101.

https://doi.org/10.4067/S0717-92002009000200005

Hechenleitner P., Gardner, M. F., Thomas, P. I., Echeverría, C., Escobar, B., Brownless, P. y Martínez, C. (2005). Plantas amenazadas del centro-sur de Chile. Universidad Austral de Chile y Real Jardín Botánico de Edimburgo. Recuperado de

http://dspace.utalca.cl/bitstream/1950/10294/1/Hechenleitner \%20\%20V.pdf

Heinrichs, S., Stiehl, C. y Müller-Using, B. (2016). Can native plant species be preserved in an anthropogenic forest landscape dominated by aliens? A case study from Mediterranean Chile. Ann. Forest Research, 59(1),75-90.

https://doi.org/10.15287/afr.2016.498

Heinrichs, S., Pauchard, A. y Schall, P. (2018). Native Plant Diversity and Composition Across a Pinus radiata D. Don Plantation Landscape in South-Central Chile-The Impact of Plantation Age, Logging Roads and Alien Species. Forest, 9(567), 1-26.

https://doi.org/10.3390/f9090567 
IPCC (Intergovernmental Panel on Climate Change) (2007). Cambio climático 2007: informe de síntesis. Contribución de los grupos de trabajo I, II y III al cuarto informe de evaluación del Crupo Intergubernamental de Expertos sobre el Cambio Climático. Ginebra, Suiza: IPCC. Recuperado de

https://www.ipcc.ch/site/assets/uploads/2018/02/ ar4_syr_sp.pdf

IUCN (International Union for Conservation of Nature and Natural Resources) (2012). The IUCN Red list of threatened species. Recuperado de

http://www.iucnredlist.org

Ladesma, M., Carranza, C. y Balzarini, M. (2010). Estimación de la biomasa foliar de Prosopis flexulosa mediante relaciones alométricas. Agriscientia, 27(2), 87-96. Recuperado de

https://revistas.unc.edu.ar/index.php/agris/article/ download/2770/2285

Le Quesne, C. y Sandoval, L. (2001). Extensión del límite sur para Nothofagaus glauca (Phil.) Krasser. Gayana Botánica, 58(2), 139-142.

https://doi.org/10.4067/S0717-66432001000200005

Lepe, V., Yuri, J. y Moggia, C. (2005). Estimación del índice de área foliar a través de la fotografía hemisférica y deshoje manual en manzanos y cerezos. Universidad de Talca. Chile. Recuperado de

http://www.sach.cl/revista/pdf/Simiente_76_3_4.pdf

Lin, J., Pan, Y., Lyub, H., Zhu, X., Li, X., Dong, B. y Li, H. (2019). Developing a two-step algorithm to estimate the leaf area index of forests with complex structures based on CHRIS/PROBA data. Forest Ecology and Management, 441(1), 57-70.

https://doi.org/10.1016/j.foreco.2019.03.030

Martínez P., G., Cellini, J. M., Peri, P. L., Lencinas, M. V., Soler E., R. y Barrera, M. (2008). Uso de fotos hemisféricas para la elaboración de modelos de altura de la regeneración en Nothofagus pumilio. $4^{\circ}$ Congreso Chileno de Ciencias Forestales, Talca, Chile. Consultado el 8 de mayo de 2019. Recuperado de

http://dspace.utalca.cl/bitstream/1950/6248/3/martinez_pastur2.pdf

Mazerolle, M. J. (2006). Improving data analysis in herpetology: using Akaike's Information Criterion (AIC) to assess the strength of biological hypotheses. Amphibia-Reptilia, 27, 169-180.

https://doi.org/10.1163/156853806777239922

Moser, G., Hertel, D. y Leuschner, C. (2007). Altitudinal change in LAI and stand leaf biomass in tropical montane forests: a transect study in Ecuador and a Pan-Tropical meta-analysis. Ecosystems, 10(6), 924-935. Recuperado de

https://www.jstor.org/stable/27823733

Muñoz, F., Cancino, J. y Rodríguez, R. (2018). Diagrama de manejo de densidad para renovales de $\mathrm{No}^{-}$ thofagus glauca (Phil.) Krasser en Chile. Madera y Bosques, 24(1), 1-9.

https://doi.org/10.21829/myb.2018.2411440

Nowak, D. (1996). Estimating leaf area and leaf biomass of open-grown deciduous urban trees. Forest Science, 42(4), 504-507. Recuperado de

https://www.nrs.fs.fed.us/pubs/jrnl/1996/ne_1996_ nowak_002.pdf

Olivas, P., Oberbauer, O., Clark, D., Ryan, M., O’Brien, J. y Ordóñez, H. (2013). Comparison of direct and indirect methods for assessing leaf area index across a tropical rainforest landscape. Agricultural and Forest Meteorology, 177, 110-116.

https://doi.org/10.1016/j.agrformet.2013.04.010

Olivares, P., San Martín, J. y Santelices, R. (2005). Ruil (Nothofagus alessandrii): estado del conocimiento y desafíos para su conservación. Talca, Chile: Departamento de Protección de Recursos Naturales, Editorial de la Comisión Nacional del Medioambiente (Conama).

Oztürk, M., Bolat, I. y Ergun, A. (2015). Influence of air-soil temperature on leaf expansion and LAI of Carpinus betulus trees in a temperate urban forest patch. Agricultural and Forest Meteorology, 200, 185-193.

https://doi.org/10.1016/j.agrformet.2014.09.014

Quiroz, I., González, M., García, E. Casanova, K. y Soto, H. (2009). Evaluación de la germinación de semillas de Nothofagus glauca (Phil.) Krasser procedentes de la Reserva Nacional Los Queules, Región del Maule. Santiago, Chile: Infor, Centro Tecnológico de la Planta Forestal. 
Rodríguez, G., González, V., Aldrete, A., de los Santos, H., Gómez, A. y Fierros, A. (2011). Modelos para estimar crecimiento y eficiencia de crecimiento en plantaciones de Pinus patula en respuesta al clareo. Revista Fitotécnia Mexicana, 34(3), 205-212. Recuperado de

http://www.scielo.org.mx/scielo.php?script=sci_abstract\&pid $=$ S0187-73802011000300012 \&lng= pt\&nrm=iso

Rojo, G., Jasso, J. y Velásquez, A. (2003). Las masas forestales como sumideros de $\mathrm{CO}_{2}$ ante un cambio climático global. Revista Chapingo, Serie Ciencias Forestales y Ambiente, 9(1), 57-67. Recuperado de https://chapingo.mx/revistas/forestales/contenido.php?section=article\&id_articulo $=388 \&$ do $i=1111$

Rubilar, R., Albaugh, T., Allen, H.L., Alvarez, J., Fox, T. y Stape, J. (2012). Influences of silvicultural manipulations on above - and belowground biomass accumulations and leaf area in young Pinus radiata plantations, at three contrasting sites in Chile. Forestry, 86, 27-38.

https://doi.org/10.1093/forestry/cps055

Ruiz, F., Murillo, B., García, J., Troyo, E., Palacios, A., Beltrán, A., Fenech, L. Zamora, S. Marrero, P., Nieto, A. y Cruz, O. (2007). Mediciones lineales en la hoja para la estimación no destructiva del área foliar en albahaca (Ocimun basilicum L.). Revista
Chapingo, Serie Horticultura, 13(1), 29-34. Recuperado de

http://www.redalyc.org/articulo.oa?id=60913104

Sillett, S. Van Pelt, R., Carroll, A., Campbell-Spickler, J., Coonen, E. y Iberle, B. (2019). Allometric equations for Sequoia sempervirens in forests of different ages. Forest Ecology and Management, 433, 349-363.

https://doi.org/10.1016/j.foreco.2018.11.016

Simonin, K., Kolb, T.E., Montes-Helu, M. y Koch, G.W. (2006). Restoration thinning and influence of tree size and leaf area to sapwood area ratio on water relations of Pinus ponderosa. Tree Physiology, 26(4), 493-503.

https://doi.org/10.1093/treephys/26.4.493

Timilsina, N., Beck, J. L., Eames, M. S., Hauer, R. y Werner, L. (2017). A comparison of local and general models of leaf area and biomass of urban trees in USA. Urban Forestry \& Urban Greening, 24, 157-163.

https://doi.org/10.1016/j.ufug.2017.04.003

Wanga, Y., Jina, G., Shib, B. y Liua, Z. (2019). Empirical models for measuring the leaf area and leaf mass across growing periods in broadleaf species with two life histories. Ecological Indicators, 102, 289-301.

https://doi.org/10.1016/j.ecolind.2019.02.041

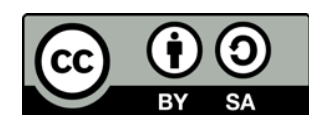

Colombia Forestal •ISSN 0120-0739 • e-ISSN 2256-201X • Bogotá-Colombia • Vol. 22 No. 2 • Julio-diciembre de 2019 • pp. 5-14 